\title{
Efficacy of a strategy to prevent neonatal early-onset group B streptococcal (GBS) sepsis
}

\author{
Regina M. Renner ${ }^{1}$, A. Renner ${ }^{2}$, Seraina \\ Schmid', Irene Hoesli', Per Nars', Wolfgang \\ Holzgreve $^{1}$ and Daniel V. Surbek ${ }^{1,3, *}$ \\ 1 Department of Obstetrics and Gynecology, University \\ Hospital, Basel, Switzerland \\ ${ }^{2}$ Department of Pediatrics, University Hospital, Berne, \\ Switzerland \\ ${ }^{3}$ Department of Obstetrics and Gynecology, University \\ Hospital Berne, Switzerland
}

\begin{abstract}
Background: Existing guidelines recommend different strategies to prevent early-onset neonatal GBS sepsis. In 1997, using our own data on incidence and risk factors, we established a new prevention strategy which includes GBS screening at 36 weeks' gestation and intrapartum antibiotic prophylaxis (IAP) in women with positive or unknown GBS colonization with at least one risk factor. The present study evaluates the efficacy of the new prevention strategy.

Methods: Retrospective study of the incidence of earlyonset GBS sepsis among all live births at the University Women's Hospital Basel between 1997 and 2002. Additional analysis of delivery and post partum period of all GBS sepsis cases, including GBS screening, risk factors during labor (prematurity, rupture of membranes (ROM) $<12 \mathrm{~h}$, intrapartum signs of infection), and IAP. Comparison of this group's characteristics G2 (9,385 live births, using the new strategy) with the previous group, G1 (1984-1993, 16,126 live births, without GBS screening or routine IAP) was performed.
\end{abstract}

Results: The incidence of early-onset GBS sepsis was reduced from 1/1000 (G1) to $0.53 / 1000$ (G2). We observed a significant reduction of overall intrapartum risk factors in cases of GBS sepsis.

Conclusion: This study suggests that our new prevention strategy is effective in reducing the incidence of early-onset GBS sepsis in neonates. In comparison, implementation of the CDC's prevention strategy might have prevented 2 additional cases in 9385 live births. However, this would have required treating a much larger

\footnotetext{
${ }^{*}$ Corresponding author:

Dr. Daniel V. Surbek

Department of Obstetrics and Gynecology

University Hospital Berne

Effingerstrasse 102

3010 Berne/Switzerland

E-mail: Daniel.Surbek@insel.ch
}

number of pregnant women with IAP with consequential increasing costs, side effects and complications.

Keywords: Antibiotic prophylaxis; early onset sepsis; female; group B streptococcus; newborn; pregnancy complications; risk factors; streptococcal infections/pc [prevention \& control]; streptococcus agalactiae; Switzerland.

\section{Introduction}

Group B streptococcus (GBS) first emerged as an important pathogen in the 1970s. Subsequently, early-onset GBS sepsis (in infants less than seven days old) became the leading cause of sepsis in newborns and one of the most common causes of neonatal infectious morbidity and mortality $[13,18]$.

At any given time, between 10 and $30 \%$ of women in the United States are colonized with GBS [18, 26]. The prevalence of colonization in Switzerland, as in other European countries, is 5-15\% [23-25]. Although GBS colonization in the gastrointestinal and genital tracts of women is most often asymptomatic, transient and does not require treatment, GBS can nevertheless cause urinary tract, skin, and soft tissue infections (including chorioamnionitis, endometritis and postpartum wound infections), or sepsis [12, 18]. During delivery, maternal vaginal colonization with GBS is an important risk factor for early-onset neonatal GBS sepsis. About half of the infants born to colonized mothers become colonized on their skin and mucosal surfaces as a result of passing through the birth canal or from GBS ascending into the amniotic fluid. One to two percent of the colonized infants will develop early-onset disease. GBS sepsis has been reported in 0.2-3.0/1000 births in the US and in some European countries [20,23]. Mortality between 4 and $10 \%$ and neurological sequelae, such as long-term hearing loss, impaired vision, and developmental problems in $10-20 \%$ are observed $[18,20,23]$.

In addition to maternal GBS carrier status, prematurity (onset of labor $<37$ completed weeks), prolonged rupture of membranes (ROM $>18 \mathrm{~h}$ after onset of labor), or intrapartum fever $\left(>100.4^{\circ} \mathrm{F} / 38^{\circ} \mathrm{C}\right)$ have been identified as risk factors for early-onset neonatal GBS sepsis. GBS bacteriuria during a current pregnancy reflects heavy colonization and is considered a risk factor, as is a previous delivery of an infant with early-onset GBS sepsis $[12,18]$. 
Table 1 Risk-based versus Screening-based approach to prevent neonatal early-onset GBS sepsis as recommended by CDC in 1996 [2].

\begin{tabular}{lll}
\hline \multirow{2}{*}{ Screening } & Risk-based & Screening-based \\
\cline { 2 - 3 } No & - Prolonged ROM $(>18 \mathrm{~h})$ & 35-37 weeks gestation \\
\hline Risk factors & - Preterm delivery $(<37$ weeks gestation $)$ & Same as in risk based \\
& - Maternal intrapartum fever $\left(>100.3^{\circ} \mathrm{F}\right.$ or $\left.37.9^{\circ} \mathrm{C}\right)$ & approach \\
& - GBS bacteriuria & If GBS positive \\
& - Previous neonate with early-onset GBS sepsis & independent of risk factor \\
IAP & - If risk factor present & If GBS unknown and \\
presence of risk factor
\end{tabular}

Since 1992, international attempts have been made to establish guidelines for preventing early-onset neonatal GBS sepsis [2, 10, 24]. Detection of maternal GBS carrier status and/or of intrapartum risk factors have been the main focus in these guidelines (Table 1). Asymptomatic colonization can be determined by culturing the bacteria from the vagina and the rectum. Culturing techniques have recently improved, and prenatal cultures taken late in pregnancy (35-37 weeks) can predict colonization status at delivery [26]. Timely detection of maternal colonization during labor is not currently feasible, given the low reliability of commercially available rapid tests [11]. Intrapartum antibiotic prophylaxis (IAP) with penicillin as the first line agent, administered to women at risk for transmitting GBS to their newborns, has been shown to decrease the risk of vertical transmission and early-onset neonatal GBS sepsis [5, 18, 22]. However, concerns related to IAP include costs, allergy (including anaphylactic reactions) and microbial resistances [9, 18, 23].

In 1995, aiming for prevention of early-onset neonatal GBS sepsis, we determined the incidence of this complication in our live births between 1984 and 1993, and evaluated the risk factors among the affected cases [24]. Based on our results, and taking into consideration above mentioned concerns, we developed a new strategy to prevent neonatal GBS sepsis, which was implemented in our department in 1997. We established screening between 35 and 37 weeks' gestation and gave IAP in GBS carriers who had an additional risk factor (rupture of membranes more than $12 \mathrm{~h}$, preterm delivery and intrapartum signs of infection). IAP was also given in case of unknown GBS colonization when another risk factor was present, or in case a previous infant had contracted GBS sepsis.

According to the revised CDC Guidelines from 2002, the benefits of a screening-based approach (general screening for GBS colonization, all women with positive culture receive IAP) outweigh the above mentioned concerns and is superior to a risk-based approach (IAP is given in the presence of risk factors without screening) [18]. The present study aimed to evaluate of the efficacy of our screening and risk-factor directed strategy.

\section{Material and Methods}

\section{Population and study design}

We conducted a retrospective analysis in order to determine the incidence of early-onset GBS sepsis among all live births at the University Women's Hospital Basel between 1997 and 2002. In addition, analysis of delivery and postpartum period of the GBS sepsis cases was performed. GBS carrier status, risk factors during labor and IAP were noted. This group (G2) of 9,385 live births had been managed by following our prevention management, implemented in 1997, which consisted of GBS screening at 35-37 weeks gestation (swab taken from the lower third of the vagina, and processed using selective broth medium), risk factor detection and administration of IAP in the presence of GBS or unknown colonization plus an additional risk factor (Table 2), or in case a previous child had contracted GBS sepsis. We further compared our findings in this group (G2) with data collected from another group of neonates (G1), born before this new prevention management had been established. G1 included 16,126 live births during the years 1984-1993. Women in G1 had not been screened for GBS and had not received routine IAP.

\section{Data collection}

Cases of early-onset GBS sepsis were identified from the data bank of the department of pediatrics as well as of the microbiology laboratory. Early-onset neonatal GBS sepsis was defined as a positive blood culture with clinical symptoms appearing within 7 days postpartum. Bacterial identification was established according to the Manual of Clinical Microbiology following NCCLS Guidelines of susceptibility testing [15]. The labor and delivery records of the affected infants and their mothers were reviewed. Summarized information for the cases included the following variables: gestational age, GBS screening, clinical risk factors (Table 2), previous infant with GBS sepsis, IAP, maternal intra- and postpartum morbidity, and neonatal mortality. The period between onset of labor or the ruptures of the membranes and delivery was defined as the "intrapartum" period.

\section{Statistical analysis}

The software of Epi Info Version 3.2 [8] was used to calculate risk ratios and their confidence intervals. For further testing of significance the Chi-square test was used, and the 2-tailed Fis- 
Table 2 Management for prevention of neonatal early-onset GBS sepsis before and after introduction of a new prevention strategy.

\begin{tabular}{lll}
\hline Group & $\begin{array}{l}\text { G1 } \\
(1984-1993,16,126 \text { live births) }\end{array}$ & $\begin{array}{l}\text { G2 } \\
(1997-2002,9,385 \text { live births) }\end{array}$ \\
\hline Management & No screening & GBS screening at 35-37 weeks' gestation \\
& No routine IAP & IAP in case of: \\
& - Positive or unknown GBS carrier \\
& status and the presence of at least \\
& one intrapartum risk factors (ROM & $>12 \mathrm{~h}$, delivery $<37$ weeks, \\
& maternal intrapartum signs of \\
& infection (temperature $\geq 38^{\circ} \mathrm{C}, \mathrm{CRP}$ \\
& $\geq 20$ mg/L, Leukocytosis $\geq 18,000 / \mu \mathrm{L}$, \\
& or persistent fetal tachycardia) \\
& - Previous neonate with early-onset \\
\end{tabular}

her's exact test was used when the expected cell value was less than 5 .

\section{Results}

The present study at the University Women's Hospital Basel evaluated the incidence of early-onset neonatal GBS sepsis in a total of 25,511 live births between the years 1984-1993 (G1, 16,126 live births) and 1997-2002 (G2, 9,385 live births). Twenty-one cases were identified, of which 16 occurred in G1, before prevention management had been implemented (Table 3). After implementation of the prevention strategy we observed 5 cases out of 9,385 live births. The incidence of early-onset GBS sepsis decreased from $1 / 1000$ (G1) to $0.53 / 1000$ live births (G2), and the Relative Risk (RR) of developing GBS sepsis in $\mathrm{G} 2$ was 0.54 times the risk observed in G1. The 95\% confidence interval $(\mathrm{Cl})$, however, included 1 (0.20-1.47), which was reflected in the statistically not significant Chi-square test $(P=0.217)$ (Figure 1).

Table 3 compares the characteristics of early-onset neonatal GBS sepsis between the two groups. As a result of establishing a management in which women with positive or unknown GBS colonization and at least one risk factor for GBS sepsis received IAP, we observed significantly fewer risk factors present during delivery of the neonates who developed GBS sepsis (Fisher exact test, $\mathrm{P}<0.001)$. Particularly, reduction of $\mathrm{ROM}<12 \mathrm{~h}$ reached statistical significance (Fisher exact test: $P=0.01$ ). The carrier status was still unknown in one of 5 term-born cases of neonatal GBS sepsis in G2 indicating incomplete implementation of screening. None of the 5 cases

Table 3 Comparison of early-onset neonatal GBS sepsis between the two groups G2 \& G1.

\begin{tabular}{|c|c|c|c|}
\hline & G1 & G2 & P-values \\
\hline Time period & 1984-1993, & $1997-2002$ & \\
\hline Live births $(n)$ & 16,126 & 9,385 & \\
\hline Cases of GBS sepsis & 16 & 5 & \\
\hline \multicolumn{4}{|c|}{ Characteristics of cases and their mothers: } \\
\hline GBS colonization in cases & $16 \times$ unknown & $\begin{array}{l}2 \times \text { positive } \\
2 \times \text { negative } \\
1 \times \text { unknown }\end{array}$ & \\
\hline Risk factors ${ }^{\star *}$ : & $15 / 16$ & $0 / 5$ & $\mathrm{P} \leq 0.001^{*}$ \\
\hline Premature delivery & $7 / 16$ & $0 / 5$ & $P=0.12$ \\
\hline Rupture of Membranes $>12 \mathrm{~h}$ & $11 / 16$ & $0 / 5$ & $P=0.01^{*}$ \\
\hline Signs of amnionitis & $7 / 16$ & $0 / 5$ & $P=0.12$ \\
\hline IAP & $0 / 16$ & $0 / 5$ & \\
\hline Mortality & $1 / 16$ & $0 / 5$ & $P=1$ \\
\hline Mean gestational age (weeks) & 37 & $40+2$ & \\
\hline $\begin{array}{l}\text { Mean interval until positive } \\
\text { blood culture (days post partum) }\end{array}$ & 1.7 & 1.6 & \\
\hline Postpartum infection & $7 / 16$ & $0 / 5$ & $P=0.12$ \\
\hline
\end{tabular}

${ }^{*}$ P-values statistically significant $(\mathrm{P}<0.05)$.

** Women may have had more than one risk factor. 
Incidence and RR of early-onset neonatal GBS disease

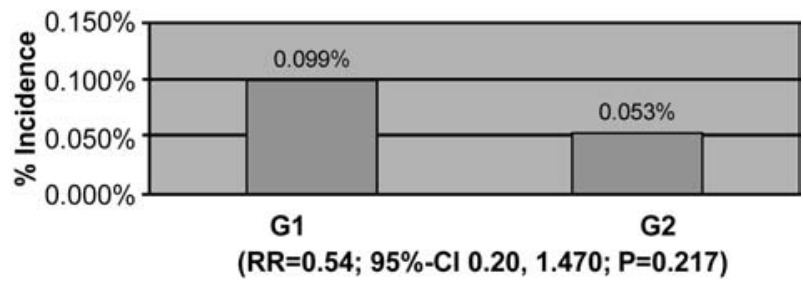

Figure 1 Incidence and RR of early-onset neonatal GBS sepsis in $\mathrm{G} 1$ compared to $\mathrm{G} 2$.

had an indication for IAP according to our management and none received IAP. The case-fatality decreased from one case, a preterm delivery, in G1 to no case in G2.

Two of the five infants who developed early-onset GBS sepsis were born to mothers who had been screened GBS positive. The first case was a male neonate, $3065 \mathrm{~g}$, born at $39+0$ weeks gestation, by vacuum extraction for bradycardia. He had good APGAR (9/10/10) scores and cord blood $\mathrm{pH}$, and initially appeared healthy. However, subsequently he developed vomiting, gray skin, and his CRP was increased. GBS was found in his blood culture four days after delivery. Antibiotic therapy resulted in rapid recovery. The second case was a male neonate, $4550 \mathrm{~g}$, born at $41+1$ weeks gestation, by vacuum extraction for macrosomia and non-reassuring fetal monitoring. Amniotic fluid contained meconium. APGAR scores were 1, 7 and 10, and cord blood pH was good. Soon after delivery, the neonate developed tachypnea and became pale. His blood culture one day later showed GBS. Antibiotic therapy with Augmentin and Fortam resulted in gradual recovery.

\section{Discussion}

Early-onset group B streptococcal sepsis has been the leading cause of neonatal sepsis and one of the most common causes for neonatal infectious mortality in the USA and Switzerland since the 1970s. Newly developed prevention strategies with intrapartum antimicrobial prophylaxis (IAP) effectively prevent the disease $[2,5,18$, 22]. However, concerns have been raised regarding costs, side effects and complications from these strategies; particularly screening and widely use of IAP $[9,18]$. The focus has been on how to best identify mothers and infants at risk for being infected and women who should receive intrapartum antibiotics.

In 1997 we implemented a new prevention strategy, which was based on our own results regarding incidence of early-onset neonatal GBS sepsis and its risk factors in 16,126 live births at our institution between 1984 and 1993 (G1) [24]. We established a screening program between 35 and 37 weeks' gestation and administered IAP in GBS carriers only if they had an additional risk factor (ROM more than $12 \mathrm{~h}$, preterm delivery and maternal intrapartum signs of infection). IAP was also given in case of unknown GBS status in the presence of a risk factor, or in case a previous child had contracted GBS sepsis (Table 2). The evaluation of 9,385 live births (G2, between 1997 and 2002) managed with our new strategy, showed a decrease in the incidence of early-onset GBS sepsis from $1 / 1000$ (G1) to $0.53 / 1000$ live births (G2). This decrease was reflected in a RR of 0.54 in G2 compared to $\mathrm{G} 1$. However, the $95 \%$ confidence interval $(\mathrm{Cl})$ included $1(0.20-1.47)$, and the $p$-value was not statistically significant $(P=0.217)$ (Figure 1). This lack of statistical significance of the result might be due to low power, a limitation often encountered in the presence of a rare disease.

Before the introduction of the US national guidelines, an incidence of 2-3 cases of early-onset neonatal GBS sepsis per 1000 live births was reported [18]. In 1996, the American College of Obstetricians and Gynecologists, the American Academy of Pediatrics, and the Centers for Disease Control and Prevention (CDC) issued guidelines for prevention, and recommended the use of either a risk-based or a screening-based approach to identify women for IAP (Table 1) [2]. Implementation of the first guidelines coincided with a $65 \%$ decline in the incidence of GBS sepsis between 1993 and 1998 (from 1.7 to $0.6 / 1000$ live births) [20].

Similar to our results, an evaluation of the 1996 Guidelines which included a large retrospective cohort study, revealed an overall incidence of $0.5 / 1000$ live births [19] Fifty-two percent of women in the cohort had been screened for GBS. Further analysis showed that the risk of GBS sepsis was significantly $(<50 \%)$ lower among infants of screened women than among those in the riskbased group (adjusted RR 0.46; $95 \% \mathrm{Cl} 0.36,0.6$ ). Projections showed that $100 \%$ implementation of the risk-based approach would have reduced the incidence from $0.5 / 1000$ to $0.44 / 1000$ live births, whereas a $100 \%$ implementation of screening-based approach would have led to a further reduction with only 0.32 infections per 1000 live births $(95 \% \mathrm{Cl} 0.26,0.38)$ [19]. Based on the evaluation, revised guidelines were published in 2002, favoring the screening-based approach [1, 3, 4, 19]. By following this screening-based approach and giving IAP to all GBS positive women, we could have potentially prevented 2 cases of sepsis and would have reduced the incidence to the $0.32 / 1000$ live births in our population (G2). This low incidence had been projected by Schrag et al. in 2002 [19] and was observed in the US in 2003 [6, 16].

Schrag et al. [19] observed that in women with a positive GBS culture but without an additional risk factor, who did not receive IAP, the incidence of disease was $1.3 / 1000$ live births $(95 \% \mathrm{Cl} 0.3,2.8)$. The higher the percentage of these women in an obstetric population, the more the overall incidence would approach 1.3/1000. 
Even though we follow the same management, our incidence of GBS sepsis is much lower than 1.3/1000 live births, probably because of the relatively lower prevalence (10-15\%) of GBS colonization in Switzerland compared to the $10-30 \%$ reported in the US [18, 23, 24].

Availability of European data about maternal GBS colonization and incidence of neonatal GBS sepsis is limited. Colonization in most European countries ranges from 10 to $20 \%$ [25]; and respectively from 10 to $15 \%$ in Swiss women [23-25]. A limitation of the present study is that it was impossible to extract the percentage of GBS screened women and the prevalence of GBS among our study population from the charts.

In accordance with our findings, early-onset GBS sepsis in Europe develops in 0.3-2/1000 live births [25]. Many European countries, including Switzerland, lack national GBS sepsis prevention guidelines, but have local protocols [25]. The German Society for Obstetrics and Gynecology has adopted the CDC guidelines for prevention of GBS sepsis in 2004, but recommend an evaluation of this management 2-3 years after its implementation [7].

Further analysis showed that in accordance with our new prevention guidelines none of our 5 GBS sepsis cases in G2 had an indication for IAP, and therefore none received IAP. This is supporting evidence for the actual implementation of our new prevention strategy, also reflected in a significant reduction of the presence of risk factors in the cases compared to cases in the previous time period (G1). GBS colonization was still unknown in one of 5 term-born cases of neonatal GBS sepsis in G2, indicating incomplete implementation of screening. Since the CDC guidelines are complex, noncompliance has been reported frequently; in up to $39 \%$ of cases with the risk-based approach and $14 \%$ with the screening-based approach. A US survey published in 2000 showed that $98 \%$ adopted a GBS policy, of which $69 \%$ used a version of a screening approach and $26 \%$ used the risk-based approach. Hospitals with any intensified prevention policy had a significant decline in incidence of early-onset disease [9, 19].

Our case-fatality decreased from one case, a preterm delivery in G1 to none in G2. Due to improved neonatal care, the survival of GBS sepsis improved in recent years (from initially 50 to now $96 \%$ ), but continues to be lower in preterm infants [5, 9].

Concerns about an optimal management are due to costs of screening and IAP, as well as adverse effects of IAP including allergic or anaphylactic reactions to IAP, emergence of antimicrobial resistant GBS strains and other pathogens, and an increased incidence of serious neonatal infections caused by pathogens other than GBS. Further issues of interest include improvement in screening and development of vaccines [9].

Cost-effective analyses in the US suggested that the risk-based approach is equally cost effective or superior to the screening-based approach [14, 17]. Rouse et al. [17] calculated an $18 \%$ maternal treatment rate in the risk-based approach, and $27 \%$ in the screening-based approach. In both strategies total costs were estimated to be similarly low. In contrast, Schrag et al. [19] showed that the anticipated intrapartum antibiotic use did not differ between the screening and risk-based strategies, when the both were implemented according to the CDC recommendations. Under screening, an estimated 30\% of deliveries received intrapartum antibiotics for any reason whereas under the risk-based approach 29\% received intrapartum antibiotics for any reason. Under each strategy $24 \%$ of deliveries had an indication for GBS prophylaxis. The authors did not present a costeffective analyses, but were in favor of the screeningbased approach, since it allowed for a broader coverage of the at-risk population, capturing colonized women without obstetric risk factors (18\% of deliveries), which resulted in a lower incidence of GBS sepsis compared to the risk-based approach [19]. We assume that by following our management we used less IAP than in either the screening-based approach favored by CDC or the pure risk-based approach, since we did not treat GBS-negative women even if presenting with risk factors, nor GBSpositive women without additional risk factor. However, in our study we could not obtain the actual frequency of IAP. Ideally, the data on GBS screening and prevalence, as well as IAP, would be collected in a prospective study. A Swiss prospective cohort study based on cost-benefit analysis came to an even more extreme conclusion. It suggested that even though both the risk- and screening-based approaches recommended by the CDC were more effective than their restrictive risk-based approach, these approaches implied increased hospital costs and a substantial increase in the proportion of women receiving antibiotics during labor, which may be unjustified in a setting of low maternal GBS carrier status. They reported an incidence of only $0.4 / 1000$ live births, while only treating $6.0 \%$ of women with antibiotics during labor. Adopting the risk-based approach as defined by the CDC they would have expected to treat $13.5 \%$ women and $16.5 \%$ with the screening-based approach [23].

Wide use of antibiotics has been criticized for several reasons. Resistance to clindamycin and erythromycin increased among GBS isolates. Concerns have also been raised regarding an emerging resistance of second line agents. Most studies find stable or declining rates of sepsis caused by other pathogens, whereas selected populations, such as preterm and very low birth weight neonates seem to be at a higher risk for sepsis caused by resistant microorganisms [9, 21]. Data on changing prevalence in resistant pathogens, i.e., E. coli, which cause illness outside the context of the labor and delivery setting, are controversial [21].

Our results showed two cases of GBS sepsis in negatively screened women. This may be explained by a pre- 
vious observation in which $15-20 \%$ of all women who had negative second trimester cultures were subsequently colonized in the late third trimester or during delivery [26]. Based on this concern, there has been a great interest in development and implementation of rapid and sensitive techniques to screen for GBS colonization and antibiotic susceptibility until effective vaccines against GBS are available for clinical use [9, 16, 21]. Vaccine development has reached phase I and II, but proceeding to phase III has turned out to be difficult, due to liability issues if tested in pregnant women [9].

In summary, our management of screening all women, but administering IAP only in women with an unknown or positive GBS culture who have an additional risk factor, intends to reduce the use of antibiotics compared to the screening-based approach suggested by the CDC. The advantage of our management versus a pure riskbased approach without screening is that IAP can be tailored to women with a positive culture and risk factors, versus treating everyone with a risk factor in the absence of screening. Thereby, we exclude many women from IAP. IAP causes more harm than screening, since in addition to costs, which results from screening as well as from IAP, IAP has adverse affects including allergic reactions and emergence of resistant pathogens. With this approach we successfully decreased our incidence of early-onset neonatal GBS sepsis to $0.53 / 1000$ live births. In order to reach the most recent reported incidence of $0.32 / 1000$ live births in the US, we would have to adopt the screening-based approach currently recommended by CDC. However, this would mean giving IAP to many more pregnant women. Our data suggest that guidelines developed for the setting present in the US should not be adopted uncritically, and that a modified strategy of the CDC guidelines can be appropriate in a setting of lower prevalence of GBS colonization. Without any doubt, development of effective rapid tests and GBS vaccines remain a priority.

\section{References}

[1] American College of Obstetricians and G: ACOG Committee Opinion: number 279, December 2002. Prevention of early-onset group B streptococcal disease in newborns. [see comment]. Obstet \& Gynecol 100 (2002) 1405

[2] Anonymous: Prevention of perinatal group B streptococcal disease: a public health perspective. Centers for Disease Control and Prevention. [erratum appears in MMWR Morb Mortal Wkly Rep 1996 Aug 9;45(31):679]. Morbidity \& Mortality Weekly Report Recommendations \& Reports 45 (1996) 1

[3] Anonymous: From the Centers for Disease Control. Revision of guidelines for the prevention of perinatal group B streptococcal disease. J Am Med Assoc 287 (2002) 1106

[4] Anonymous: ACNM Clinical Bullletin. Early-onset group B strep infection in newborns: Prevention and prophylaxis number 2, April 2003. J Midwifery \& Women's Health 48 (2003) 375

[5] Boyer KM, SP Gotoff: Prevention of early-onset neonatal group B streptococcal disease with selective intrapartum chemoprophylaxis. New England J Med 314 (1986) 1665

[6] Centers for Disease Control and P: Diminishing racial disparities in early-onset neonatal group B streptococcal disease - United States, 2000-2003. MMWR Morbidity \& Mortality Weekly Report 53 (2004) 502

[7] DGGG. Empfehlungen zur Prophylaxe der Neugeborenensepsis (fruehe Form) durch Streptokokken der Gruppe B. (2004). http://www.dggg.de/leitlinien/pdf/4-3-4.pdf

[8] Epi Info ${ }^{\mathrm{TM}}$. 2004; Centers for Disease Control and Prevention (CDC)

[9] Gibbs RS, S Schrag, A Schuchat: Perinatal infections due to group B streptococci. Obstet \& Gynecol 104 (2004) 1062

[10] Gilbert R: Prenatal screening for group B streptococcal infection: gaps in the evidence. Int J Epidemiol 33 (2003) 2

[11] Hickman ME, MA Rench, P Ferrieri, CJ Baker: Changing epidemiology of group B streptococcal colonization. Pediatrics 104 (1999) 203

[12] Jolivet RR, P Centers for Disease Control and: Early-onset neonatal group B streptococcal infection: 2002 guidelines for prevention. J Midwifery \& Women's Health 47 (2002) 435

[13] McCracken G: Group B streptococci: the new challenge in neonatal infections. J Pediatr 82 (1973) 703

[14] Mohle-Boetani JC, A Schuchat, BD Plikaytis, JD Smith, CV Broome: Comparison of prevention strategies for neonatal group B streptococcal infection. A population-based economic analysis. J Am Med Assoc 270 (1993) 1442

[15] NCCLS: Manual of Clinical Microbiology. (2005)

[16] Puopolo KM, LC Madoff, EC Eichenwald: Early-Onset Group B Streptococcal Disease in the Era of Maternal Screening. Pediatrics 115 (2005) 1240

[17] Rouse DJ, RL Goldenberg, SP Cliver, GR Cutter, ST Mennemeyer, CA Fargason, Jr: Strategies for the prevention of early-onset neonatal group B streptococcal sepsis: a decision analysis. Obstet \& Gynecol 83 (1994) 483

[18] Schrag S, R Gorwitz, K Fultz-Butts, A Schuchat: Prevention of perinatal group B streptococcal disease. Revised guidelines from CDC. Morbidity \& Mortality Weekly Report. Recommendations \& Reports 51 (2002) 1

[19] Schrag SJ, ER Zell, R Lynfield, A Roome, KE Arnold, AS Craig, LH Harrison, A Reingold, K Stefonek, G Smith, M Gamble, A Schuchat, T Active Bacterial Core Surveillance: A population-based comparison of strategies to prevent early-onset group B streptococcal disease in neonates. [see comment]. New England J Med 347 (2002) 233

[20] Schrag SJ, S Zywicki, MM Farley, AL Reingold, LH Harrison, LB Lefkowitz, JL Hadler, R Danila, PR Cieslak, A Schuchat: Group B streptococcal disease in the era of intrapartum antibiotic prophylaxis. [see comment]. New England J Med $342(2000) 15$

[21] Schuchat A: Impact of intrapartum chemoprophylaxis on neonatal sepsis. Pediat Infect Disease J 22 (2003) 1087

[22] Smaill F: Intrapartum antibiotics for group B streptococcal colonisation. Cochrane Database of Systematic Reviews (2000) CD000115

[23] Stan CM, M Boulvain, PA Bovier, R Auckenthaler, M Berner, O Irion: Choosing a strategy to prevent neonatal early-onset group B streptococcal sepsis: economic evaluation. BJOG: and Internat J Obstet \& Gynaecol 108 (2001) 840

[24] Surbek DS, J Pavic, N Almendral, AC: Neonatale Streptokokken-B Sepsis: Izidenz und Riskofaktoren. Arch Gynecol Obstet 257 (1995) 148 
[25] Trijbels-Smeulders MA, LA Kollee, AH Adriaanse, JL Kimpen, LJ Gerards: Neonatal group B streptococcal infection: incidence and strategies for prevention in Europe. [see comment]. Pediat Infect Disease J 23 (2004) 172

[26] Yancey MK, A Schuchat, LK Brown, VL Ventura, GR Markenson: The accuracy of late antenatal screening cultures in predicting genital group B streptococcal colonization at delivery. Obstet \& Gynecol 88 (1996) 811

Received July 8, 2005. Revised August 29, 2005. Accepted October 10, 2004. 